\title{
Friction and Diffusion of Matter-Wave Bright Solitons
}

\author{
Subhasis Sinha, ${ }^{1}$ Alexander Yu. Cherny, ${ }^{1,2}$ Dmitry Kovrizhin, ${ }^{1,3}$ and Joachim Brand ${ }^{1}$ \\ ${ }^{1}$ Max Planck Institute for the Physics of Complex Systems, Nöthnitzer Straße 38, 01187 Dresden, Germany \\ ${ }^{2}$ Bogoliubov Laboratory of Theoretical Physics, Joint Institute for Nuclear Research, 141980, Dubna, Russia \\ ${ }^{3}$ RRC Kurchatov Institute, Kurchatov Sq. 1, 123182 Moscow, Russia
}

(Received 9 June 2005; published 26 January 2006)

\begin{abstract}
We consider the motion of a matter-wave bright soliton under the influence of a cloud of thermal particles. In the ideal one-dimensional system, the scattering process of the quasiparticles with the soliton is reflectionless; however, the quasiparticles acquire a phase shift. In the realistic system of a BoseEinstein condensate confined in a tight waveguide trap, the transverse degrees of freedom generate an extra nonlinearity in the system which gives rise to finite reflection and leads to dissipative motion of the soliton. We calculate the velocity and temperature-dependent frictional force and diffusion coefficient of a matter-wave bright soliton immersed in a thermal cloud.
\end{abstract}

DOI: 10.1103/PhysRevLett.96.030406

PACS numbers: 03.75.Pp, 05.60.Gg, 42.65.Tg

Solitons are localized waves that propagate without spreading and attenuation. They appear from classical systems like ocean waves to optics and quantum systems like Bose-Einstein condensates (BEC) of atomic gases. A $\mathrm{BEC}$ of a dilute atomic gas with attractive two-body interactions in three dimensions (3D) is unstable and collapses [1]. In one dimension (1D), however, a BEC with attractive interaction is stable against collapse and forms a selfbound particlelike object known as a bright soliton. Recently, BEC bright solitons were observed in quasi-1D waveguide traps at Rice University [2], at ENS in Paris [3], and at the University of Heidelberg [4].

One of the most important features of solitons is the nondispersive motion over long distances [5]. BEC solitons, moreover, cannot bind thermal excitations [6] and thus may separate from the cloud of thermal atoms that is always present in BEC experiments. Because of these properties, BEC solitons may find applications in quantum interferometry experiments. Matter-wave interferometers with BECs are expected to boost the precision measurement of inertial forces by several orders of magnitude as compared to present-day technology with optical lasers or cold atoms. Thus it is very important to understand to which extent the interactions of a BEC with a thermal cloud can affect the precision of interferometric measurements. At the same time, a quantitative understanding of thermal effects may also allow us to precisely measure temperatures in the $\mathrm{nK}$ regime.

In this Letter we discuss how dissipative effects in the motion of a soliton in a thermal cloud can arise due to the 3D nature of the BEC in a tight waveguide. We consider the scattering of quasiparticles by the quasi-1D matterwave soliton [7], taking account of the transverse degrees of freedom. Muryshev et al. considered the interaction of quasiparticles with dark BEC solitons and found that these lead to acceleration and eventually disintegration of the soliton in a thermal environment [8]. Following a similar line of arguments, we show that the quasiparticles scatter- ing on a bright soliton have a finite probability of reflection only due to the transverse extent of the soliton, which finally gives rise to dissipative effects. The bright soliton experiences friction and diffusive motion in a thermal cloud but maintains its integrity in contrast to dark solitons which disintegrate. Diffusive processes in optical solitons due to a different mechanism have already been studied in Ref. [9].

A BEC in a waveguide with a harmonic transverse confinement is well described by the Gross-Pitaevskii (GP) equation:

$$
i \hbar \frac{\partial \psi}{\partial t}=\left[-\frac{\hbar^{2}}{2 m} \nabla^{2}+\frac{1}{2} m \omega^{2} \rho^{2}+\frac{4 \pi \hbar^{2} a}{m}|\psi|^{2}\right] \psi,
$$

where $\psi$ is the macroscopic wave function of the condensate, $\omega$ is the frequency of transverse trapping potential, and $a$ is the 3D scattering length. In the quasi-1D limit the effective dynamics of the system takes place along the free axis ( $x$ axis) without exciting the transverse modes. The quasi-1D limit can be achieved when the mean field interaction is smaller than the radial excitation frequency, $4 \pi \hbar^{2}|a||\psi|^{2} / m<\hbar \omega$. Aiming at an adiabatic separation of slow longitudinal and fast transverse motion we can write the full 3D wave function assuming cylindrical symmetry as $\psi(\vec{r}, t)=\phi(x, t) \chi(\rho, x, t)$. Here, $\phi$ is the 1D (longitudinal) wave function and $\chi$ is the radial wave function with the normalization convention $\int|\chi|^{2} 2 \pi \rho d \rho=1$ and $\int|\phi|^{2} d x=N$, where $N$ is the number of bosons in the system. In the adiabatic approximation we now assume that the radial wave function $\chi$ weakly depends on the slow variables $x$ and $t$ and the derivatives of $\chi$ can be neglected. After substituting the ansatz for the wave function into Eq. (1), we obtain the following adiabatically decoupled equations for the longitudinal and the transverse wave functions:

$$
i \hbar \partial_{t} \phi=-\frac{\hbar^{2}}{2 m} \partial_{x}^{2} \phi+\tilde{\mu} \phi,
$$




$$
\left[-\frac{\hbar^{2}}{2 m} \nabla_{\rho}^{2}+\frac{1}{2} m \omega^{2} \rho^{2}+\frac{4 \pi \hbar^{2} a}{m} n|\chi|^{2}\right] \chi=\tilde{\mu} \chi,
$$

where we have introduced the transverse chemical potential $\tilde{\mu}$, which has to be found from the ground state solution of Eq. (3) as a function of the linear density $n(x, t)=$ $|\phi(x, t)|^{2}$. Physical solutions of Eq. (3) are found only if - an $<0.47$ [10], otherwise transverse collapse occurs [11]. In the following we will be interested in the quasi$1 \mathrm{D}$ regime of small an and expand $\tilde{\mu}($ an $)$ in a power series.

In the quasi-1D limit, when $|a| n \ll 0.47$, the radial wave function $\chi$ will be close to the ground state of the 2D harmonic oscillator with a Gaussian profile. We can expand $\chi$ in terms of the radial eigenmodes $\varphi_{\nu}(\rho)$, $\chi(\rho, x)=\varphi_{0}(\rho)+\sum_{\nu} C_{\nu}(x) \varphi_{\nu}(\rho)$. The coefficients $C_{\nu}$ are small and can be calculated perturbatively. The transverse chemical potential $\tilde{\mu}$ can be obtained by using perturbation theory: $\tilde{\mu}=\hbar \omega+g n-g_{2} n^{2}+\cdots$, where $g=2 a \hbar \omega$ and $g_{2}=24 \ln (4 / 3) a^{2} \hbar \omega$. The constant $g_{2}$ was calculated first in Ref. [8]. Corrections to the coupling constants $g$ and $g_{2}$, beyond the GP approach presented here have been found in Ref. [12]. We obtain the following effective equation describing the condensate in the quasi1D limit:

$$
\left[-\left(\hbar^{2} / 2 m\right) \partial_{x}^{2}+g|\phi|^{2}-g_{2}|\phi|^{4}\right] \phi=\mu \phi .
$$

This is a nonlinear Schrödinger equation with a cubic and a quintic nonlinearity, as used before in Ref. [8]. An estimate from the 3D GP equation (1) gives stability of a single soliton solution if $N|a| / l<0.627$ is fulfilled [13].

Without the extra nonlinearity associated with $g_{2}$, Eq. (4) is integrable. For attractive interactions at $a<0$, the bosons form a self-bound particlelike state known as a bright soliton with the wave function $\phi(x)=$ $\sqrt{N / 2 b} \operatorname{sech}(x / b)$ and the chemical potential $\mu=$ $-\hbar^{2} / 2 m b^{2}$, where $b=l^{2} /(N|a|)$. We notice that for a weak soliton parameter $N|a| / l \lesssim 1$, the system becomes quasi one dimensional $(b \geq l)$.

A soliton can be considered as a macroscopic particle of mass $m N$, moving in the bath of thermal excitations. Dissipative motion of the soliton arises due to the scattering of thermal atoms. Here we consider the interaction of thermally excited particles with the soliton within the Bogoliubov formalism [7],

$$
\left[H_{0}+H_{1}\right] \psi=\epsilon(k) \psi,
$$

where $\psi=(u, v)$ is a two component vector of particle $(u)$ and hole $(v)$ amplitudes, and $\epsilon$ is the quasiparticle energy. The unperturbed Hamiltonian $H_{0}$ and the perturbation $H_{1}$ are given by

$$
\begin{gathered}
\hat{H}_{0}=\left(\begin{array}{cc}
-\frac{\hbar^{2}}{2 m} \partial_{x}^{2}-\mu & 0 \\
0 & +\frac{\hbar^{2}}{2 m} \partial_{x}^{2}+\mu
\end{array}\right) \\
\hat{H}_{1}=\left(\begin{array}{cc}
V_{1}(x) & V_{2}(x) \\
-V_{2}^{*}(x) & -V_{1}(x)
\end{array}\right),
\end{gathered}
$$

where $\quad V_{1}=2 g|\phi|^{2}-3 g_{2}|\phi|^{4} \quad$ and $\quad V_{2}=g \phi^{2}-$ $2 g_{2}|\phi|^{2} \phi^{2}$. The scattering states have energy $\boldsymbol{\epsilon}(k)=$ $\frac{\hbar^{2} k^{2}}{2 m}+|\mu|$. In one dimension, neglecting the extra nonlinearity $\left(g_{2}=0\right)$, we obtain the exact solution of the scattering states:

$$
\begin{gathered}
u_{k}=A(k)[k b+i \tanh (x / b)]^{2} e^{i k x} \\
v_{k}=A(k) \operatorname{sech}^{2}(x / b) e^{i k x},
\end{gathered}
$$

where $A(k)=1 /\left(k^{2} b^{2}-1\right)$ is a normalization constant. The transmittance is given by

$$
t=(k b+i)^{2} /(k b-i)^{2},
$$

and the transmission probability is $|t|^{2}=1$. Hence, the quasiparticles scatter without reflection on the soliton but only acquire a phase shift in the scattering process. Reflectionless scattering on a soliton in the integrable nonlinear Schrödinger equation (4) (with $g_{2}=0$ ) is a wellknown result of mathematical soliton theory and is also found in an exact solution of the quantum many-body model in the limit of large particle number [6]. In the quasi-1D limit, the soliton thus becomes transparent and exhibits dissipationless motion in a thermal cloud.

Now we consider the scattering problem of quasiparticles in the presence of an extra nonlinearity. This nonlinear term breaks the integrability and leads to a finite reflection probability. For sufficiently small linear particle density $n \ll|a|$, we can take the coupling constant $g_{2}$ as a small parameter and solve the scattering problem using Green's function techniques. In order to solve Eq. (5) for the particle amplitude $u$ we construct the Green's function for the $u$ component, which is given by $G_{1}\left(x-x^{\prime}\right)=$ $\left(m / \hbar^{2} k\right) \sin \left(k\left|x-x^{\prime}\right|\right)$. Since the potential is symmetric, the scattering states can be taken obeying even or odd symmetry. The Lippmann-Schwinger equation for the particle channel can be written as

$$
\begin{aligned}
u_{e / o}= & u_{e / o}^{0}+\int G_{1}\left(x-x^{\prime}\right) V_{1}\left(x^{\prime}\right) u_{e / o}\left(x^{\prime}\right) d x^{\prime} \\
& +\int G_{1}\left(x-x^{\prime}\right) V_{2}\left(x^{\prime}\right) v_{e / o}\left(x^{\prime}\right) d x^{\prime},
\end{aligned}
$$

where $u_{e / o}$ denotes even [odd] wave functions of the particle states and $u_{e}^{0}=\cos (k x),\left[u_{o}^{0}=\sin (k x)\right]$. The most general wave function has the form: $u_{k}=$ $A u_{k}^{e}+B u_{k}^{o}$. Asymptotically this wave function becomes $\lim _{x \rightarrow-\infty} u_{k}=e^{i k x}+r e^{-i k x}$ and $\lim _{x \rightarrow \infty} u_{k}=t e^{i k x}$, where $|t|^{2}$ and $R=|r|^{2}$ are the transmission and the reflection coefficient, respectively. We obtain $R(k)$ by solving Eqs. (4) and (5) numerically and matching with the asymptotic solutions, see Fig. 1.

An analytical estimate of the reflection coefficient can be obtained from Eq. (11) by approximating $\phi=$ $\sqrt{N / 2 b} \operatorname{sech}(x / b)$ and $u_{e / o}$ and $v_{e / o}$ with the properly symmetrized solutions (8) and (9). This becomes exact for $g_{2}=0$ and relies on $g_{2}$ being a small parameter. The 


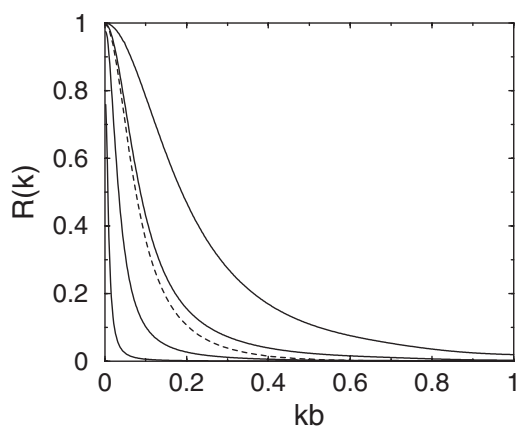

FIG. 1. Reflection coefficient of a soliton as a function of momentum, for $N|a| / l=0.1,0.2,0.3,0.4$ (from smaller to larger values of reflection coefficient). The dotted line shows the analytical estimate (12) for $N|a| / l=0.2$.

reflection coefficient is given by $R=|r|^{2}$ and

$$
r(k)=-i \frac{I_{+}+I_{-}}{\left(I_{+}-i\right)\left(I_{-}+i\right)},
$$

where the terms $I_{+}$and $I_{-}$are

$$
I_{ \pm}=\mp 2 A(k)\left[k b+6 \ln (4 / 3) \frac{N^{2}|a|^{2}}{l^{2}} \frac{Q_{ \pm}(k b)}{k b}\right]
$$

with $Q_{ \pm}(x)=1 / 3+x^{2} \pm\left(1+x^{2}\right)^{2} \pi x /[3 \sinh (\pi x)]$. The Lippmann-Schwinger formalism gives the correct limiting behavior for small $k$ where $R \rightarrow 1$, in contrast to a simple Born approximation. Total reflection is expected whenever extra nonlinearity breaks the special resonant conditions leading to reflectionless scattering at $k=0$, as generically found in one-dimensional scattering [14]. This case is very different from phonons scattering on a perturbed dark soliton, which becomes transparent for small $k$ as found in Ref. [8]. This anomalous scattering behavior arises from the presence of a Goldstone mode at $\epsilon=0$ governing the long wavelength $(k=0)$ phonon scattering, even in the presence of perturbations in the nonlinearity. For bright solitons, on the contrary, the Goldstone modes at $\epsilon=0$ are separated by an energy gap of $|\mu|$ from the scattering continuum at $\epsilon(k)=\frac{\hbar^{2} k^{2}}{2 m}+|\mu|$ and therefore do not influence the scattering at $k=0$. The approximation (12) reproduces the qualitative features but slightly overestimates the exact values of $R$ as seen in Fig. 1.

The reflection coefficient $R$ is a function of dimensionless momentum $k b$ and the soliton parameter $N|a| / l$. Once we know the interaction of particles with a soliton from the microscopic theory, we can describe its motion in the bath of thermal particles at a given temperature. Because of its large mass $m N$, we may treat the soliton as a classical particle. The thermal atoms impart a momentum to the soliton in the scattering process. While the soliton is at rest, the force on the soliton cancels on the average but, nevertheless, the stochastic nature of the force introduces a diffusive motion of the soliton. For a moving soliton, the average force imparted by the thermal particles does not vanish and gives rise to a frictional force on the soliton. To include the dissipative effects in the soliton's motion we write down the kinetic equation for the phase space distribution function $f(p, q, t)$ of the soliton's center of mass coordinate:

$$
\frac{\partial f}{\partial t}-\frac{\partial}{\partial p}\left(\frac{\partial H}{\partial q} f\right)+\frac{\partial}{\partial q}\left(\frac{\partial H}{\partial p} f\right)=I_{\text {coll }},
$$

where, for small momentum transfer, the collision integral can be written as: $I_{\text {coll }}=\frac{\partial}{\partial p}\left[A f+\frac{\partial}{\partial p}(B f)\right]$. The terms $A$ and $B$ give rise to friction and diffusion of the soliton, respectively. The frictional force $A$ can be computed from the following expression,

$$
A=\int \frac{d k}{2 \pi}(-2 \hbar k) R(k)\left|\frac{\partial \epsilon(k)}{\hbar \partial k}\right| N\left(E, k_{B} T\right),
$$

where $N\left(E, k_{B} T\right)$ describes the distribution of thermal particles in the frame of the moving soliton with velocity $v$ and the energy $E$ takes the value $E(k)=(\hbar k-$ $m v)^{2} / 2 m$. In each collision, the particle with momentum $k$ has a probability $R$ to reflect back and transfer the momentum $-2 \hbar k$ to the soliton. This momentum transfer multiplied with the number of particles coming from each direction per unit time gives rise to a frictional force. When the soliton is at rest, the momentum transfer on each direction cancels on the average and as a result the friction vanishes.

At finite temperatures the thermal atoms are distributed according to the rules of quantum statistics. Although thermal equilibrium may be reached in an external trap [15], the subtle conditions of equilibrium are not necessarily fulfilled in a dynamical experimental situation. Here, we consider the motion of a soliton relative to a significantly warmer thermal cloud of atoms. We thus can assume that the thermal cloud obeys a classical Boltzmann distribution $N\left(E, k_{B} T\right) \sim \exp \left(-E / k_{B} T\right)$. Dissipative effects on the soliton are stronger with increasing density or increasing temperature of the thermal cloud. We consider the situation where $10^{4}$ thermal particles are confined within a length $L=50 b(\approx 70 \mu \mathrm{m}$ for ENS soliton with $b=$ $1.4 \mu \mathrm{m}$ ), with a density of the thermal gas of $n b=200$ $\left(\approx 10^{12} / \mathrm{cm}^{3}\right)$ and the velocity distribution of the thermal particles being controlled by changing the temperature. Within a certain range of the soliton velocity the frictional force increases linearly with velocity as seen in Fig. 2. When the velocity is increased further, nonlinear effects take over and the force decreases.

The diffusion parameter of the transport equation reads

$$
B=\int \frac{d k}{2 \pi} 2(\hbar k)^{2} R(k)\left|\frac{\partial \epsilon(k)}{\hbar \partial k}\right| N\left(E, k_{B} T\right) .
$$

This term describes the velocity fluctuations of the soliton and gives rise to a diffusion in the momentum space. A graph is shown in Fig. 3.

So far we considered only the low-energy elastic scattering of thermal quasiparticles on the soliton. To restrict our discussion to the quasi-1D case, we neglected the 

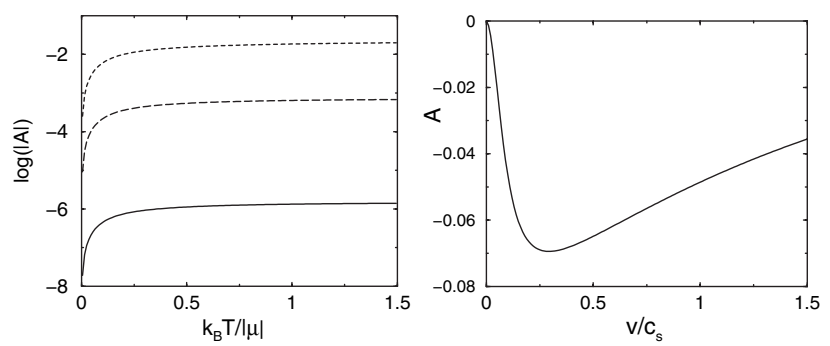

FIG. 2. (a) Logarithm of the friction force $A$ in units of $\hbar \omega / l$ as a function of temperature for soliton velocity $V=0.1 \mathrm{~h} / \mathrm{mb}$, density of thermal gas $n b=200$, and soliton parameter $N|a| / l=0.2$ (solid line), 0.3 (dashed line), 0.4 (dotted line). (b) Friction force as a function of velocity, for $N|a| / l=0.3$, $k_{B} T /|\mu|=0.5$ [units and parameters as Fig. 2(a)].

higher energy radial excitations $\sim \hbar \omega$. As an additional effect, the soliton can radiate particles if the colliding quasiparticle has higher energy than the binding energy $|\mu|$. Also nonlinear collective motion of the thermal cloud and the soliton is possible [16]. However, the elastic scattering process discussed in this work will dominate if the condition $k_{B} T<|\mu|<\hbar \omega$ is fulfilled. A tight radial trapping potential is suitable to avoid inelastic scattering processes. In the ENS experiment [3], the oscillator length of radial confinement was $l=1.4 \mu \mathrm{m}$. For a soliton parameter $N|a| / l=0.4$, the sound velocity at the center of the soliton becomes $c_{\mathrm{s}} \approx 2.5 \mathrm{~mm} / \mathrm{s}$. If a soliton with $N \sim 10^{3}$ particles moves with a velocity $0.1 c_{\mathrm{s}}$, then it decelerates $5.01 \mathrm{~mm} / \mathrm{s}^{2}$ due to the frictional force. Finally it stops after $0.05 \mathrm{~s}$, traveling a distance of $6 \mu \mathrm{m}$. The slowing down of a bright soliton can thus be observed experimentally in a realistic parameter regime.

For small velocities, $A=\gamma v$ and the moving soliton stops after a time scale $\tau=m N / \gamma$. Because of the diffusion process the energy of a resting soliton changes as $E=$ $(B / 2 \gamma)\left[1-e^{-2 \gamma t / m N}\right]$. For $t \ll \tau$, the energy of the soliton increases and finally it reaches a steady state with energy $E=B / 2 \gamma$. Bright solitons immersed in a thermal bath can provide an ideal system to study thermally activated tunneling over a barrier [17] and to study the dynamics of soliton trains at finite temperature [2], where dissipative motion may result in soliton fusion.

In conclusion, we have investigated the effects of a thermal environment on the dynamics of bright matterwave solitons and have calculated the frictional force and diffusion coefficient in a microscopic approach. Friction and diffusion effects occur due to the deviation from the quasi-one-dimensional limit. Both effects can be controlled by the parameters of the system and can be made small if unattenuated propagation of solitons is desired. Thus bright solitons are very interesting for future applications in high precision quantum interferometry experiments. On the other hand, the parameters can be chosen such that the dissipative effects become accessible to experimental observation with currently available tech-

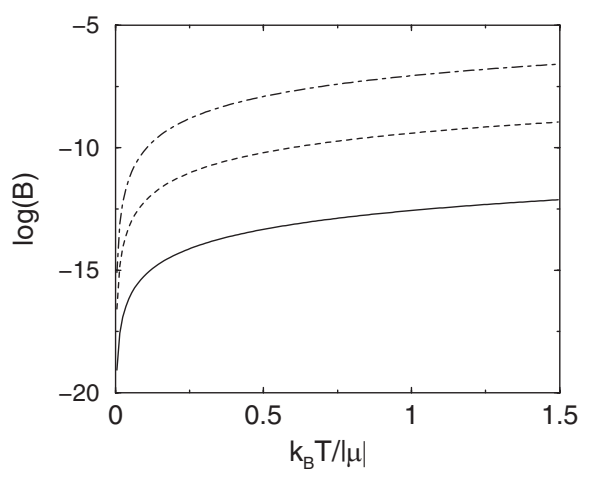

FIG. 3. Logarithm of the diffusion coefficient $B$ (in units of $\hbar m \omega^{2}$ ) of a soliton with zero average velocity as a function of temperature for the soliton parameters $N|a| / l=0.2$ (solid line), 0.3 (dashed line), 0.4 (dot-dashed line), and a density of the thermal gas of $n b=200$.

niques, e.g., to study the dynamics of a multisoliton system in a thermal environment.

We acknowledge enlightening discussions with G. Shlyapnikov who suggested this problem.

[1] J. M. Gerton, D. Strekalov, I. Prodan, and R. G. Hulet, Nature (London) 408, 692 (2000).

[2] K. E. Strecker, G. B. Partridge, A. G. Truscott, and R. G. Hulet, Nature (London) 417, 150 (2002).

[3] L. Khaykovich et al., Science 296, 1290 (2002).

[4] B. Eiermann et al., Phys. Rev. Lett. 92, 230401 (2004).

[5] Y. Kivshar, Optical Solitons (Academic Press, London, 2003).

[6] J. B. McGuire, J. Math. Phys. (N.Y.) 5, 622 (1964).

[7] A. Wynveen et al., Phys. Rev. A 62, 023602 (2000); U. V. Poulsen and K. Mølmer, ibid. 67, 013610 (2003); J. Brand, I. Häring, and J.-M. Rost, Phys. Rev. Lett. 91, 070403 (2003); S. Flach et al., Phys. Rev. Lett. 90, 084101 (2003).

[8] A. E. Muryshev et al., Phys. Rev. Lett. 89, 110401 (2002).

[9] P.D. Drummond, R. M. Shelby, S. R. Friberg, and Y. Yamamoto, Nature (London) 365, 307 (1993); J.F. Corney and P.D. Drummond, J. Opt. Soc. Am. B 18, 153 (2001).

[10] L. Bergé, T. J. Alexander, and Y. S. Kivshar, Phys. Rev. A 62, 023607 (2000); M. I. Weinstein, Commun. Math. Phys. 87, 567 (1983).

[11] L. D. Carr and J. Brand, Phys. Rev. Lett. 92, 040401 (2004); Phys. Rev. A 70, 033607 (2004).

[12] M. Olshanii, Phys. Rev. Lett. 81, 938 (1998); A. Y. Cherny and J. Brand, Phys. Rev. A 70, 043622 (2004).

[13] L. D. Carr and Y. Castin, Phys. Rev. A 66, 063602 (2002).

[14] M. Sassoli de Bianchi, J. Math. Phys. (N.Y.) 35, 2719 (1994).

[15] V. Dunjko, C. P. Herzog, Y. Castin, and M. Olshanii, condmat/0306514.

[16] H. Buljan, M. Segev, and A. Vardi, Phys. Rev. Lett. 95, 180401 (2005).

[17] L. I. McCann, M. Dykman, and B. Golding, Nature (London) 402, 785 (1999). 